\title{
Gene Regulatory Network Analysis Defines Transcriptome Landscape with Alternative Splicing of Human Umbilical Vein Endothelial Cells during Replicative Senescence
}

\section{Momoko Ohori}

Takeda Pharmaceutical Company

Yusuke Nakayama

Takeda Pharmaceutical Company

Mari Shimizu-Ogasawara

Takeda Pharmaceutical Company

Hiroyoshi Toyoshiba

Takeda Pharmaceutical Company

Atsushi Nakanishi

Takeda Pharmaceutical Company

Samuel Aparicio

BC Cancer Agency

Shinsuke Araki ( $\square$ shinsuke.araki@takeda.com )

Takeda Pharmaceutical Company

\section{Research Article}

Keywords: replicative senescence, HUVEC, alternative splicing, microexon, MYC, SIRT2

Posted Date: January 12th, 2021

DOl: https://doi.org/10.21203/rs.3.rs-140983/v1

License: @ (i) This work is licensed under a Creative Commons Attribution 4.0 International License.

Read Full License

Version of Record: A version of this preprint was published at BMC Genomics on December 1st, 2021. See the published version at https://doi.org/10.1186/s12864-021-08185-x. 


\section{Abstract}

\section{Background}

Endothelial cell senescence is the state of permanent cell cycle arrest and plays a critical role in the pathogenesis of age-related diseases. However, a comprehensive understanding of the gene regulatory network, including genome-wide alternative splicing machinery, involved in endothelial cell senescence is lacking.

\section{Results}

We thoroughly described the transcriptome landscape of replicative senescent human umbilical vein endothelial cells. Genes showing a monotonic increase or decrease in expression with the culture period were defined as hub genes in the co-expression network. Computational network analysis of these genes led to the identification of canonical and non-canonical senescence pathways, such as MYC and SIRT2 signaling, which were down-regulated in metabolism and chromosome organization processes pathways. Additionally, we showed that endothelial cell senescence involves alternative splicing. Importantly, the first and last exon types of splicing, as observed in FLT1 and ACACA, were preferentially altered among the alternatively spliced genes during endothelial senescence. We further identified novel microexons in PRUNE2 and $P S A P$, each containing $9 \mathrm{nt}$, which were altered within the specific domain during endothelial senescence.

\section{Conclusions}

These findings unveil the comprehensive transcriptome pathway and novel signaling regulated by RNA processing, including gene expression and splicing, in replicative endothelial senescence.

\section{Introduction}

Cellular senescence is a permanent state of cell cycle arrest caused by the interruption of cell division with limited replicative capacity, also referred to as replicative senescence [1, 2]. Besides cell cycle arrest, senescent cells exhibit morphological changes including the formation of an enlarged body mass and the up-regulation of senescence-associated $\beta$-galactosidase (SA- $\beta$-Gal) activity. Multi-signaling pathways induce and maintain senescence, which is characterized by DNA damage, oxidative stress, and telomere shortening [2]. All senescence-associated signaling pathways converge at the activation of cyclindependent kinase inhibitors p16 (CDKN2A), p15 (CDKN2B), p21 (CDKN1A), and p27 (CDKN1B), which are commonly used as molecular markers for senescence [3-5]. Senescent cells accumulate in aged tissues, leading to age-related diseases, such as cancer, cardiovascular disease, diabetes, and neurodegenerative disorders [6, 7]. Endothelial cell senescence is considered a key mechanism of the induction of agerelated vascular diseases, such as coronary artery disease, stroke, and hypertension, through vascular endothelial dysfunction [8]. Hence, exploring the signaling pathway and mechanism of endothelial cell 
senescence is critical for developing disease treatment strategies. The role of oxidative stress and mitochondrial dynamics in endothelial cell senescence has been reported previously $[9,10]$.

Global transcriptome analysis enables a comprehensive understanding of complex biological processes. Therefore, this technique has been used in several studies to identify senescence-related gene expression changes in inflammatory and mitochondrial pathways in fibroblasts [11-13]. Additionally, alternative splicing (AS) of genes, such as p53, SIRT1, and IGF1, has gained attention as a key determinant of cellular differentiation and aging-associated senescence $[14,15]$. However, our current knowledge of the gene regulatory network including genome-wide splicing involved in replicative endothelial cell senescence is limited.

In this study, we conducted a detailed computational transcriptome analysis of senescence-induced human umbilical vein endothelial cells (HUVECs). We identified canonical and non-canonical transcriptional responses induced by cellular senescence in HUVECs compared with those induced by senescence in fibroblasts. Besides gene expression analysis, we clarified the characteristics of AS variants in HUVEC senescence. Our results showed that alternative first exon (AFE) and alternative last exon (ALE) types of splicing were predominantly induced by HUVEC senescence. Further investigation revealed that microexons, which encode small proteins of 1-17 amino acids, are highly evolutionarily conserved and play important roles in various cellular functions and diseases with tissue specificity [16]. Moreover, RNA-seq analysis revealed the alteration of novel microexons during HUVEC senescence. Overall, this study elucidated the transcriptome pathway and novel signaling in HUVEC senescence.

\section{Results}

\section{Replicative senescence of HUVECs}

We prepared senescent HUVECs, which ceased to proliferate, in the presence of a complete culture medium for comprehensive transcriptome analysis. After 60 days of culture, four independent HUVECs (C1-4) stopped dividing, which showed that the population doubling (PD) rate was $<0.1$ per day (Fig. $1 \mathrm{~A})$. Approximately $70 \%-80 \%$ of the cells showed SA- $\beta$-Gal activity, and the cell size was 3.4 -fold greater on day 60 than it was on day 22 (Fig. 1B,C). The expression of CDKN2A was induced 10-fold greater on days 73 and 74 than it was on days 5-7 (Fig. 1D). These cellular phenotypes were characteristic of replicative senescent cells, consistent with a previous report (Salama et al., 2014).

\section{Comparative transcriptomic analysis of senescent HUVECs and fibroblasts}

To investigate transcriptome responses during endothelial cellular senescence, we conducted RNA-seq analysis of two independent HUVEC clones (C1 and C2), with four different culture periods $(5,18 / 20$, $60 / 61$, and 74 days) (Fig. 2A). First, to compare the gene expression signature with the previous data set 
of senescence-related genes, we conducted hypergeometric test-based enrichment analysis of senescence-related gene sets extracted from three different databases, namely, Molecular Signatures Database (MSigDB), REACTOME, and Human Ageing Genomic Resources (HAGR) [17-19]. Genes showing differential expression between days 5 and 74 (fold change $>2$ ) were significantly enriched among the known senescence-related gene sets (Fig. 2B). The most significantly enriched gene set of "FRIDMAN_SENESCENCE_UP" was previously reported to be up-regulated in senescent cells (C1, $P<1 \times 10^{-9} ; C 2, P<1 \times 10^{-4}$ ) [20]. The gene set of the p53-mediated pathway and senescenceassociated secretory phenotype was enriched among genes both up- and down-regulated in senescent cells (up-regulated genes: $\mathrm{C} 1$ and $\mathrm{C} 2, P<0.005$; down-regulated genes: $\mathrm{C} 1, P<0.05 ; \mathrm{C} 2, P<0.0005$ ) and up-regulated in senescent $\mathrm{C} 1$ and $\mathrm{C} 2$ clones $\left(\mathrm{C} 1, P<1 \times 10^{-4} ; \mathrm{C} 2, P<0.05\right)$. Other senescence-related gene sets did not show significant enrichment with genes showing expression changes during HUVEC senescence. These results suggest that the signature expression pattern of some genes is common to senescent HUVECs and fibroblasts, and the remaining genes are involved in a distinct senescence-related pathway in HUVECs.

Next, we examined the distribution of differentially expressed genes (DEGs) and the alternatively spliced genes (ASGs) between young (day 5) and senescent (day 74) HUVECs. The distributions of fragments per kilobase of exon model per million reads mapped (FPKM) in young and senescent HUVECs are shown in Fig. 2C. ASGs were quantified by MISO analysis, with variation in percent spliced-in ( $\triangle \mathrm{PSI}$ ) (Bayes score $>10$ ) as shown in Fig. 2D. Because AS occurs in various biological processes, especially cellular differentiation [21], we analyzed DEGs and ASGs during neuronal differentiation from induced pluripotent stem cells (iPSCs) for 10 days [22]. The results showed a three-fold change in FPKM values, thus providing 1222, 1520, and 3227 genes corresponding to C1, C2, and iPSCs, respectively (Fig. 2E). The magnitude of DEGs during senescence was statistically greater than that during neuronal differentiation from iPSCs (mean values: C1, 3.91; C2, 3.80; iPSCs, 3.64 as a scale of log2), as determined by Wilcoxon tests $(C 1, P=0.04 ; C 2, P=0.0004)$. Additionally, 637, 451, and 1561 genes were identified in $\mathrm{C} 1, \mathrm{C} 2$, and iPSC data sets, respectively, using the thresholds of $\triangle P S I>0.1$ and Bayes factor $>10$, and the magnitude of $\triangle P S I$ in senescent clones was smaller than that in the iPSC data set (mean values: $\mathrm{C} 1,0.25 ; \mathrm{C} 2,0.27$; iPSCs, 0.32), as determined by the Wilcoxon test (C1, $\left.P<1 \times 10^{-22} ; \mathrm{C} 2, P<1 \times 10^{-9}\right)$ (Fig. 2F). These results suggest that the distribution of DEGs was greater than that of the neuronal differentiation of iPSCs and the pattern of ASGs in senescent clones was smaller than that in iPSCs. This motivated us to conduct a more detailed computational analysis of gene expression and AS.

\section{Key regulators indicating monotonic changes in FPKM during HUVEC senescence}

To examine the biological significance of responsive transcripts in senescent HUVECs, we conducted Weighted Correlation Network Analysis (WGCNA) of the FPKM transcript data. We identified four dominant FPKM profile modules (turquoise, blue, brown, and yellow) containing $60.6 \%$ of the clustered 
peak events among 20 different modules (Figs $3 \mathrm{~A}, \mathrm{~S} 1$, and S2). These four dominant modules showed a monotonic increase or decrease in responses with the culture period, whereas the other modules showed nonmonotonic patterns in a small number of genes, implying that nonmonotonic patterns detected secondary effects during senescence (Fig. 3A). Next, we conducted a functional enrichment analysis of each set of four dominant modules to identify the pathways affected by senescence. Only modules with decreasing FPKM (turquoise and blue) showed significant enrichment of biological processes such as cell cycle, DNA repair, metabolism, transcription, translation, splicing, and chromosome organization, as determined using the hypergeometric test $\left(P<1 \times 10^{-4}\right)$ (Fig. 3B, Table S1). Among these, metabolism and chromosome organization processes were down-regulated in both the turquoise and blue modules, implying that key molecules for cellular senescence are included in these processes.

Next, we analyzed the regulatory network of each of the four dominant modules by determining which genes were involved in the regulation of genes in each module. Genes with high connectivity $(>0.8)$ were defined as hub genes; these genes were considered as key genes in a co-expression network [23]. A hub gene has the potential to affect many genes in its network. We identified 963, 247, 163, and 179 hub genes in the turquoise, blue, brown, and yellow modules, respectively (Fig. S3). To predict the transcriptional regulatory network of hub genes, we conducted a computational analysis using two different databases: the ingenuity pathway analysis (IPA) upstream database and transcription factor binding site (TFBS) database [24, 25]. The IPA upstream regulator analysis revealed potential upstream regulators of genes in each module, and upstream gene analysis using TFBSs in MSigDB revealed upstream gene candidates for each module (Fig. $3 C$ ). Canonical senescence-related molecules, identified as upstream regulators such as $E 2 F 1, E 2 F 4, R B 1, C D K N 1 A, C D K N 2 A$, and $M Y C$, were included in the turquoise module. Particularly, $M Y C$ included in the blue module was identified as an up-regulator in both turquoise and blue modules, suggesting that $M Y C$ is a key regulator of not only HUVEC senescence but also fibroblast senescence. Interestingly, the sirtuin (SIRT) family, SIRT2 and SIRT6, was identified as an up-regulator of genes in the blue module. SIRT2 was included in the yellow module as a gene showing a monotonic increase in expression. SIRT1 is a well-known senescence-related SIRT family protein; however, the role of SIRT2 in senescence remains unclear. Taken together, WGCNA and upstream pathway analysis suggest that canonical and non-canonical senescent pathways, such as MYC and SIRT2 signaling pathways, regulate HUVEC senescence.

\section{Predominant changes in AFE- and ALE-type splicing during HUVEC senescence}

To examine how HUVEC senescence affects AS, we quantitated the AS events between senescent HUVECs at early (day 5) and late (days 60-74) timepoints during culture (Fig. 4A). We classified the

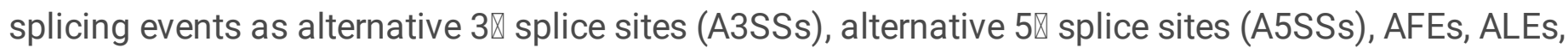
mutually exclusive exons (MXEs), retained introns (RIs), and skipped exons (SEs) (Fig. 4A,B). The proportion of AFE- and ALE-type splicing increased after HUVEC senescence, and the fold increase in the number of AFEs and ALEs was greater than that in the number of SEs in both $\mathrm{C} 1$ and $\mathrm{C} 2$ clones during 
senescence (AFE: 3.1- and 1.5-fold; ALE: 1.8- and 3.1-fold; SE: 1.6- and 1.2-fold, respectively). Among the alternatively spliced events between day 5 and day 74 in HUVEC culture, 74 splicing events overlapped between $\mathrm{C} 1$ and $\mathrm{C} 2$ clones in a statistically significant manner, as determined by Fisher's exact test $\left(P=8.96 \times 10^{-72}\right)$, suggesting that the identified ASGs depend on HUVEC senescence, not reflecting the difference between $\mathrm{C} 1$ and $\mathrm{C} 2$ (Fig. 4C). Some of the overlapped splicing events were detected in three senescence-related genes (EFEMP1, FLT1, and TCF3) (Fig. 4D, Table S2). A3SS-, ALE-, and SE-type splicing was altered during senescence in EFEMP1, FLT1, and TCF3, respectively. Among the ASGs, except senescence-related genes, the $\triangle \mathrm{PSI}$ distribution for the AFE-type splicing of ACACA (acetyl-CoA carboxylase-a $[\mathrm{ACCa}]$ ) was significantly altered in both $\mathrm{C} 1$ and $\mathrm{C} 2$ clones during senescence (Bayes factor $>10^{12}$ ) (Fig. 4E). Multiple promoters regulate ACCa mRNA, registered as NM_198836 and NM_198834, in the presence of thyroid hormone, glucagon, and medium-chain fatty acids [26] (Fig. 4F). Quantitative PCR analysis revealed that the expression of NM_198836 was decreased compared with that of NM_198834 in a time-dependent manner during senescence, implying that AFE-type splicing of ACCa mRNA is involved in HUVEC senescence (Fig. 4G). Taken together, splicing alterations in senescence- and non-senescence-related genes were induced during HUVEC senescence, and AFE- and ALE-type splicing was predominantly altered compared with other types of splicing.

\section{Microexon alterations during HUVEC senescence}

Besides canonical splicing analysis, we examined whether microexons are induced by HUVEC senescence using VAST-TOOLS, which detects splicing alterations at all hypothetical splice junctions formed by the usage of annotated and unannotated splice sites [27]. The results of VAST-TOOLS analysis were similar to those of MISO analysis; however, more RI events were detected because of the increased sensitivity for unannotated splice sites (Fig. 5A). We identified several microexon alterations during HUVEC senescence, although to a lesser extent (Table S3). Among these, two microexons in PRUNE2 and $P S A P$, each containing $9 \mathrm{nt}$, were altered in both $\mathrm{C} 1$ and $\mathrm{C} 2$ clones during senescence. To confirm these results, we amplified the microexon-containing region from each isoform by reverse transcription PCR (RTPCR). Besides $\mathrm{C} 1$ and $\mathrm{C} 2$ clones, we examined clones $\mathrm{C} 3$ and C4. Consistent with VAST-TOOLS results, microexons in PRUNE2 decreased and microexons in PSAP increased the PSI value, with statistical significance or tendency, during HUVEC senescence in all clones (Fig. 5B). Each altered microexon contained the CRAL-TRIO lipid-binding domain and saposin B-type domain in PRUNE2 and PSAP, respectively, implying that microexon alteration affects the function of the encoded protein.

Furthermore, to examine whether readthrough transcripts were induced by HUVEC senescence, we conducted an RNA-seq analysis using deFuse [28]. The readthrough transcript refers to a conjoined gene arising from the upstream transcript to the downstream transcript of partner genes with poly $(A)$ sites [29, 30]. However, RNA-seq data showed that the number of conjoined genes did not change, indicating that HUVEC senescence does not involve conjoined genes (Fig. 5C). Together, our results suggest that a limited number of microexon splicing alterations are induced during HUVEC senescence. 


\section{Discussion}

To clarify the transcriptome landscape of HUVECs during replicative senescence, we analyzed changes in gene expression and genome-wide splicing using comprehensive multi-technical RNA-seq analysis. Genes showing a monotonic increase or decrease in expression were identified as hub genes. Additionally, canonical and non-canonical senescence pathways were identified during HUVEC senescence. Canonical senescence-related molecules, including $M Y C$, E2F family, RB1, CDKN1A (p21), and $C D K N 2 A$ (p16), were identified from the turquoise module as upstream regulators. Generally, $M Y C$ inhibits two inhibitory regulators, CDKN1A (yellow module) and CDKN2A (brown module), and subsequently activates the E2F and RB1 pathways of turquoise and blue modules, respectively (Fig. 6). $M Y C$ inactivation is also known to induce global changes in chromatin structure and epigenetic modifications (reduction in histone $\mathrm{H} 4$ acetylation and increase in histone $\mathrm{H} 3$ lysine 9 [H3K9] methylation) [31]. Additionally, total E2F reduction induces senescence-like cell cycle arrest in cancer cells [32]; particularly, E2F1 blocks cell proliferation [33]. Given that chromatin assembly is one of the hub functions of down-regulated biological functions (Fig. 3B), MYC and E2F pathways could play key roles in the down-regulation of genes related to cell cycle, transcription, splicing, and translation during HUVEC senescence.

Besides the canonical pathway during senescence, SIRT2 was identified as one of the genes with monotonically increasing expression in the yellow module and concomitantly identified as an upregulator in the blue module. The SIRT family proteins function as NAD ${ }^{+}$-dependent histone deacetylases and master regulators of metabolism and aging [34]. SIRT1 and SIRT6 are well-known to regulate HUVEC senescence via the regulation of transcription factors, p53, E2F1, and nuclear factor-kappa B $[35,36]$. However, the role of SIRT2 in senescence has not been well elucidated, although it is known that SIRT2 is involved in the stress-induced premature senescence, but not in the quiescence, of U2OS osteosarcoma cell line [37]. The time-dependent up-regulation of SIRT2 during HUVEC senescence suggests the involvement of a novel SIRT2-related signaling pathway during HUVEC senescence, although further investigation is needed to confirm whether SIRT2 acts as a trigger of HUVEC senescence.

Novel splicing alterations during HUVEC senescence were also investigated in this study, although the distribution of ASGs in senescent clones was smaller than the neuronal differentiation of iPSCs. Particularly, AFE- and ALE-type splicing was predominantly induced by senescence. Interestingly, hub genes identified during HUVEC senescence included key splicing factors, such as SRSF1, SRSF6, hnRNPAO, and $h n R N P A 1$, among others (Table S1). Two independent large-scale microarray-based expression cohorts in human blood have revealed that the expression of key splicing factors, SRSFs and hnRNPs, varies with age [38]. Additionally, changes in the expression of splicing factor genes is correlated with cognitive decline and longevity in humans and mice [39]. Presumably, gene expression changes of splicing factors during senescence are involved in splicing alteration and translation, thereby contributing to aging and longevity. We further identified that novel microexon alterations are involved in HUVEC senescence, albeit to a lesser extent. These evidence raise the possibility that induced splicing isoforms preform distinct and cooperative functions during senescence and impact senescence signaling. 


\section{Conclusion}

Transcriptional regulatory analysis, based on co-expressed gene clusters, during HUVEC senescence clarified hub genes as well as canonical and non-canonical responses, including MYC and SIRT2 signaling, which were down-regulated in metabolism and chromosome organization processes. Besides changes in the expression of canonical splicing factor genes, novel splicing alterations such as AFE- and ALE-type splicing and microexons were identified. Our findings define a comprehensive transcriptome network, including gene expression and splicing, in the complex mechanisms of replicative endothelial senescence.

\section{Materials And Methods Cell culture}

HUVECs (Lonza, Basel, Switzerland) were cultured using an EBM-2 Bullet Kit (Lonza) and passaged until reaching senescence. The PD rate was calculated using the following equation [40]:

$$
P D=\frac{\ln (\text { No. of cells harvested })-\ln (\text { No.of cells seeded })}{\ln 2}
$$

Then, HUVECs were subcultured at $37^{\circ} \mathrm{C}$ in an atmosphere containing $5 \% \mathrm{CO}_{2}$.

\section{SA- $\beta$-Gal staining}

Cell fixation and SA- $\beta-$ Gal staining were conducted using Cellular Senescence Assay Kit (CBA-230) according to the manufacturer's instructions (Cell Biolabs, Inc., San Diego, CA, USA). Briefly, cells were fixed with Fixing Solution for 5 min at room temperature, washed with phosphate-buffered saline, and then stained with Cell Staining Working Solution overnight at $37^{\circ} \mathrm{C}$ in the dark.

\section{RNA-seq analysis}

To comprehensively investigate transcriptome consequences during HUVEC senescence, two different HUVEC clones of HUVECs (C1 and C2) cultured for four different durations were analyzed by RNA-seq. The four timepoints selected for RNA-seq analysis included day 5 or 6 (youngest timepoint), day 18 or 20 (dividing phase), day 60 or 61 (when HUVECs stopped dividing; PD $<0.1$ per day), and day 74 (oldest timepoint; 2 weeks after HUVECs stopped dividing). RNA-seq libraries were aligned to the hg19 (GRCh37) reference genome assembly using the Genomic Short-read Nucleotide Alignment Program (GSNAP) [41, 42]. Next, the mate-pair information of the aligned libraries was fixed, potential PCR duplicates were removed, and libraries were sorted using SAMtools [43]. Then, gene expression was quantified (as FPKM 
values) using Cufflinks [44]. Differential splicing was detected using MISO analysis [45] and VAST-TOOLS [27]. In MISO analysis, the following parameters were used for removing AS events: Bayes factor $<10$, variation in percent spliced-in $(|\triangle P S| \mid)<0.1$, and zero reads supporting the inclusion or exclusion isoform or less than 10 reads supporting either of the event isoforms. Conjoined genes (readthrough transcripts) were identified using the deFuse software (omicX, France) [28], with the classifier modified by removing the est_breakseqs_percident and breakseqs_estislands_percident features since the inclusion of these classifier features can decrease the probability of gene fusion calls. Conjoined genes were identified by selecting deFuse gene fusion calls, where both participating genes were located on the same strand of the same chromosome, using the following parameters: deletion $=$ " $Y$ "; splice score $=4$; exonboundaries $=$ " $\mathrm{Y}$ "; probability $\geq 0.9$.

RNA-seq data of neuronal differentiation in human iPSCs were obtained from the Gene Expression Omnibus database (GSE32625) [22]. The raw sequence data were aligned to the hg19 reference genome using GSNAP and analyzed using Cufflinks and MISO analysis (as described above).

\section{Co-expression network analysis}

Gene expression response profiles were clustered using the WGCNA R software [23]. Soft-threshold selection was facilitated by calculating the scale-free network topology model fit $R^{2}$ values for soft thresholds 1-30 using the pickSoftThreshold function. The final threshold value was manually selected, where the topology model fit was both relatively stable and high.

Genes with FPKM $\geq 1$ in at least one sample of the $\mathrm{C} 1$ and $\mathrm{C} 2$ RNA-seq data sets were selected for clustering. Gene expression profiles were clustered using WGCNA R with the following parameters: networkType $=$ "signed"; minModuleSize $=30$. Genes in the RNA-seq data sets of both $\mathrm{C} 1$ and $\mathrm{C} 2$ clones were classified into 20 co-expression clusters using the following parameters: soft power threshold $=12$; deepSplit $=0$.

\section{Biological process enrichment analysis and enrichment map generation}

To determine whether the expression of known senescence-related genes is altered during HUVEC senescence, enrichment analysis was conducted using senescence gene sets extracted from MSigDB, REACTOME, and HAGR databases, as well as genes of both HUVEC clones (C1 and C2) showing differential expression between the youngest timepoint (day 5) and oldest timepoint (day 74).

Gene Ontology (GO) biological process term enrichment for a set of genes was conducted by generating functional interaction networks with the BiNGO Cytoscape plug-in [46] using genes in the resulting network. The GO terms were selected using the following thresholds: $P=1.0 \mathrm{e}^{-7}$; false discovery 
rate $=1.0 \mathrm{e}^{-3}$; overlap coefficient $=0.6$. Enrichment maps were generated for the BiNGO biological process enrichment results using the EnrichmentMap Cytoscape plug-in [47].

\section{Transcriptional regulatory analysis}

Transcriptional regulatory factors were predicted using upstream analysis with the IPA database (Qiagen, Qiagen Redwood City, CA, USA; www.qiagen.com/ingenuity) and enrichment analysis with the TFBS database. To conduct the TFBS enrichment analysis, the MSigDB compendium of transcription factor target gene sets was used. The enrichment significance was evaluated by hypergeometric statistics [18].

\section{Hub gene analysis}

Genes with high intramodular connectivity ( $k$ Within $>0.8$ ) were defined as hub genes. The kWithin, which was calculated using WGCNA, represents the scaled degree of connectivity of the edge of the gene under the same module.

\section{RT-PCR}

Total RNA was extracted using the RNeasy Plus Mini kit (Qiagen), and CDNA was synthesized using the SuperScript VILO cDNA Synthesis Kit (Life Technologies Corporation, CA, USA) or the PrimeScript RT reagent Kit (Takara Bio Inc., Japan), according to the manufacturers' instructions. Real-time PCR was conducted using TaqMan MGB probe (Life Technologies), and the amount of FAM fluorescence was measured as a function of the PCR cycle number $\left(\mathrm{C}_{\mathrm{T}}\right)$ using the Applied Biosystems ViiA7 Real-Time PCR system (Life Technologies). To examine microexon alterations, primers were designed to contain alternative exons using Primer3 (http://bioinfo.ut.ee/primer3-0.4.0/). RT-PCR was conducted using AmpliTaq Gold (Life Technologies) using the following conditions: an initial denaturation step at $95^{\circ} \mathrm{C}$ for $5 \mathrm{~min}$, followed by 35 cycles at $94^{\circ} \mathrm{C}$ for $30 \mathrm{~s}, 60^{\circ} \mathrm{C}$ for $30 \mathrm{~s}$, and $72^{\circ} \mathrm{C}$ for $45 \mathrm{~s}$, and a final elongation step at $72^{\circ} \mathrm{C}$ for $7 \mathrm{~min}$. The PCR products were separated by capillary electrophoresis, and signal intensity was measured using the LabChip GX Touch Nucleic Acid Analyzer (PerkinElmer, Inc., MA, USA). Hs01060665_g1 (Life Technologies) for $\beta$-actin, Hs00923894_m1 (Life Technologies) for CDKN2A, and the following primers and probes were used for amplification: ACACA39440 (probe): 5'ATCGCCCGACCGCACACGTTGC-3'; ACACA39440-F: 5'-GCACGCCTGTCAGCCATC-3'; ACACA39440-R: 5'TCCACTTCCAGAAAGACCTCAG-3'; ACACA35313 (probe): 5'-CACCACATCCTCTCATCATTGCGCCTCA-3'; ACACA35313-F: 5'-GGTGAAGAGGGTGCGTTTCA-3'; ACACA35313-R: 5'-

CCCTCAAGATTGACATCAGAGTAGA-3'; PSAP-F: 5'-TTGCTATCCAGATGATGATGC-3'; PSAP-R: 5'CCTCATCACAGAACCCAACC-3'; PRUNE2-F: 5'-GTCATCGAGCCCTACAGGAG-3'; PRUNE2-R: 5'GCATTTAGACCGTCCCCATA-3'.

\section{Abbreviations}


SA- $\beta$-Gal: Senescence-associated $\beta$-galactosidase, AS: Alternative splicing, HUVEC: Human umbilical vein endothelial cell, AFE: Alternative first exon, ALE: Alternative last exon, MSigDB: Molecular Signatures Database, HAGR: Human Ageing Genomic Resources, DEG: Differentially expressed gene, ASG: Alternatively spliced gene, FPKM: Fragments per kilobase of exon model per million reads mapped, iPSC: Induced pluripotent stem cell, WGCNA: Weighted Correlation Network Analysis, IPA: Ingenuity pathway analysis, TFBS: Transcription factor binding site, A3SS: Alternative 3囚 splice site, A5SS: Alternative 5囚 splice site, MXE: Mutually exclusive exon, RI: Retained intron, SE: Skipped exon, RT-PCR: Reverse transcription PCR, GSNAP: Genomic Short-read Nucleotide Alignment Program, GO: Gene Ontology

\section{Declarations}

\section{Ethics approval and consent to participate}

Not applicable.

\section{Consent for publication}

Not applicable.

\section{Availability of data and materials}

RNA-seq raw sequencing data are available at GEO (GEO series accession number: GSE163251) and all data generated or analyzed during this study are included in this published article and its supplementary information files.

\section{Competing interests}

M.O., Y.N., M.S.O., H.T., A.N., and S.Ar are/were employees of Takeda Pharmaceutical Company Limited. S.A. has no any competing interests.

\section{Funding}

Takeda Pharmaceutical Company Limited provided support in the form of salaries for authors but did not have any additional role in the study design, data collection and analysis, decision to publish, or preparation of the manuscript.

\section{Authors' contributions}


M.O., Y.N., and S.Ar designed the study and wrote the manuscript. M.O. performed the experiments. Y.N. performed RNA-seq data analysis with assistance from M.S.O.. H.T., A.N. and S.A supervised the project.

\section{Acknowledgements}

We thank O. Nakanishi and R. Hara for the great support for this study. We thank T. Ogasawara, H. Kato Y. Goto for technical assistance. We thank K. Nishimura for the great discussion. We thank all the members of the Shonan incubation laboratory and BC cancer agency members for great encouragement and support for our research.

\section{References}

1. Muñoz-Espín D, Serrano M: Cellular senescence: from physiology to pathology. Nature reviews Molecular cell biology 2014, 15(7):482-496.

2. Kuilman T, Michaloglou C, Mooi WJ, Peeper DS: The essence of senescence. Genes Dev 2010, 24(22):2463-2479.

3. Kurz DJ, Decary S, Hong Y, Erusalimsky JD: Senescence-associated (beta)-galactosidase reflects an increase in lysosomal mass during replicative ageing of human endothelial cells. J Cell Sci 2000, 113 (Pt 20):3613-3622.

4. Chen QM: Replicative senescence and oxidant-induced premature senescence. Beyond the control of cell cycle checkpoints. Ann N Y Acad Sci 2000, 908:111-125.

5. Salama R, Sadaie M, Hoare M, Narita M: Cellular senescence and its effector programs. Genes Dev 2014, 28(2):99-114.

6. McHugh D, Gil J: Senescence and aging: Causes, consequences, and therapeutic avenues. J Cell Biol 2018, 217(1):65-77.

7. Hernandez-Segura A, Nehme J, Demaria M: Hallmarks of Cellular Senescence. Trends in cell biology 2018, 28(6):436-453.

8. Donato AJ, Morgan RG, Walker AE, Lesniewski LA: Cellular and molecular biology of aging endothelial cells. J Mol Cell Cardiol 2015, 89(Pt B):122-135.

9. Meijles DN, Sahoo S, Al Ghouleh I, Amaral JH, Bienes-Martinez R, Knupp HE, Attaran S, Sembrat JC, Nouraie SM, Rojas MM et al: The matricellular protein TSP1 promotes human and mouse endothelial cell senescence through CD47 and Nox1. Sci Signal 2017, 10(501).

10. Kim YM, Youn SW, Sudhahar V, Das A, Chandhri R, Cuervo Grajal H, Kweon J, Leanhart S, He L, Toth PT et al: Redox Regulation of Mitochondrial Fission Protein Drp1 by Protein Disulfide Isomerase Limits Endothelial Senescence. Cell Rep 2018, 23(12):3565-3578.

11. Purcell M, Kruger A, Tainsky MA: Gene expression profiling of replicative and induced senescence. Cell Cycle 2014, 13(24):3927-3937. 
12. Marthandan S, Baumgart M, Priebe S, Groth M, Schaer J, Kaether C, Guthke R, Cellerino A, Platzer M, Diekmann S et al: Conserved Senescence Associated Genes and Pathways in Primary Human Fibroblasts Detected by RNA-Seq. PLoS One 2016, 11(5):e0154531.

13. Bar-Joseph Z, Gitter A, Simon I: Studying and modelling dynamic biological processes using timeseries gene expression data. Nat Rev Genet 2012, 13(8):552-564.

14. Deschênes $M$, Chabot $B$ : The emerging role of alternative splicing in senescence and aging. Aging Cell 2017, 16(5):918-933.

15. Rodríguez SA, Grochová D, McKenna T, Borate B, Trivedi NS, Erdos MR, Eriksson M: Global genome splicing analysis reveals an increased number of alternatively spliced genes with aging. Aging Cell 2016, 15(2):267-278.

16. Irimia M, Weatheritt RJ, Ellis JD, Parikshak NN, Gonatopoulos-Pournatzis T, Babor M, QuesnelVallieres $M$, Tapial J, Raj B, O'Hanlon D et al: A highly conserved program of neuronal microexons is misregulated in autistic brains. Cel/ 2014, 159(7):1511-1523.

17. Fabregat A, Jupe S, Matthews L, Sidiropoulos K, Gillespie M, Garapati P, Haw R, Jassal B, Korninger F, May B et al: The Reactome Pathway Knowledgebase. Nucleic Acids Res 2018, 46(D1):D649-d655.

18. Subramanian A, Tamayo P, Mootha VK, Mukherjee S, Ebert BL, Gillette MA, Paulovich A, Pomeroy SL, Golub TR, Lander ES et al: Gene set enrichment analysis: a knowledge-based approach for interpreting genome-wide expression profiles. Proc Natl Acad Sci U S A 2005, 102(43):15545-15550.

19. Tacutu R, Thornton D, Johnson E, Budovsky A, Barardo D, Craig T, Diana E, Lehmann G, Toren D, Wang $J$ et al: Human Ageing Genomic Resources: new and updated databases. Nucleic Acids Res 2018, 46(D1):D1083-d1090.

20. Fridman AL, Tainsky MA: Critical pathways in cellular senescence and immortalization revealed by gene expression profiling. Oncogene 2008, 27(46):5975-5987.

21. He L, Bai $Q$, Tang $\mathrm{L}$ : Alternative splicing regulates pluripotent state in pluripotent stem cells. Curr Stem Cell Res Ther 2015, 10(2):159-165.

22. Lin M, Pedrosa E, Shah A, Hrabovsky A, Maqbool S, Zheng D, Lachman HM: RNA-Seq of human neurons derived from iPS cells reveals candidate long non-coding RNAs involved in neurogenesis and neuropsychiatric disorders. PLoS One 2011, 6(9):e23356.

23. Langfelder $P$, Horvath S: WGCNA: an R package for weighted correlation network analysis. $B M C$ Bioinformatics 2008, 9:559.

24. Kramer A, Green J, Pollard J, Jr., Tugendreich S: Causal analysis approaches in Ingenuity Pathway Analysis. Bioinformatics 2014, 30(4):523-530.

25. Veerla S, Ringner M, Hoglund M: Genome-wide transcription factor binding site/promoter databases for the analysis of gene sets and co-occurrence of transcription factor binding motifs. $B M C$ genomics 2010, 11:145.

26. Yin L, Zhang Y, Charron T, Hillgartner FB: Thyroid hormone, glucagon, and medium-chain fatty acids regulate transcription initiated from promoter 1 and promoter 2 of the acetyl-CoA carboxylase-alpha gene in chick embryo hepatocytes. Biochimica et biophysica acta 2000, 1517(1):91-99. 
27. Tapial J, Ha KCH, Sterne-Weiler T, Gohr A, Braunschweig U, Hermoso-Pulido A, Quesnel-Vallieres M, Permanyer J, Sodaei R, Marquez $Y$ et al: An atlas of alternative splicing profiles and functional associations reveals new regulatory programs and genes that simultaneously express multiple major isoforms. Genome Res 2017, 27(10):1759-1768.

28. McPherson A, Hormozdiari F, Zayed A, Giuliany R, Ha G, Sun MG, Griffith M, Heravi Moussavi A, Senz $\mathrm{J}$, Melnyk $\mathrm{N}$ et al: deFuse: an algorithm for gene fusion discovery in tumor RNA-Seq data. PLoS Comput Biol 2011, 7(5):e1001138.

29. Prakash T, Sharma VK, Adati N, Ozawa R, Kumar N, Nishida Y, Fujikake T, Takeda T, Taylor TD: Expression of conjoined genes: another mechanism for gene regulation in eukaryotes. PLOS One 2010, 5(10):e13284.

30. Akiva P, Toporik A, Edelheit S, Peretz Y, Diber A, Shemesh R, Novik A, Sorek R: Transcription-mediated gene fusion in the human genome. Genome Res 2006, 16(1):30-36.

31. Wu CH, van Riggelen J, Yetil A, Fan AC, Bachireddy $\mathrm{P}$, Felsher DW: Cellular senescence is an important mechanism of tumor regression upon c-Myc inactivation. Proc Natl Acad Sci U S A 2007, 104(32):13028-13033.

32. Maehara K, Yamakoshi K, Ohtani N, Kubo Y, Takahashi A, Arase S, Jones N, Hara E: Reduction of total E2F/DP activity induces senescence-like cell cycle arrest in cancer cells lacking functional pRB and p53. J Cell Biol 2005, 168(4):553-560.

33. Wu L, Timmers C, Maiti B, Saavedra HI, Sang L, Chong GT, Nuckolls F, Giangrande P, Wright FA, Field SJ et al: The E2F1-3 transcription factors are essential for cellular proliferation. Nature 2001, 414(6862):457-462.

34. Chang HC, Guarente L: SIRT1 and other sirtuins in metabolism. Trends in endocrinology and metabolism: TEM 2014, 25(3):138-145.

35. Cardus A, Uryga AK, Walters G, Erusalimsky JD: SIRT6 protects human endothelial cells from DNA damage, telomere dysfunction, and senescence. Cardiovascular research 2013, 97(3):571-579.

36. Brooks CL, Gu W: How does SIRT1 affect metabolism, senescence and cancer? Nat Rev Cancer 2009, 9(2):123-128.

37. Anwar T, Khosla S, Ramakrishna G: Increased expression of SIRT2 is a novel marker of cellular senescence and is dependent on wild type p53 status. Cell Cycle 2016, 15(14):1883-1897.

38. Holly AC, Melzer D, Pilling LC, Fellows AC, Tanaka T, Ferrucci L, Harries LW: Changes in splicing factor expression are associated with advancing age in man. Mech Ageing Dev 2013, 134(9):356-366.

39. Bhadra M, Howell P, Dutta S, Heintz C, Mair WB: Alternative splicing in aging and longevity. Human genetics 2020, 139(3):357-369.

40. van der Loo B, Fenton MJ, Erusalimsky JD: Cytochemical detection of a senescence-associated betagalactosidase in endothelial and smooth muscle cells from human and rabbit blood vessels. Exp Cell Res 1998, 241(2):309-315.

41. Wu TD, Watanabe CK: GMAP: a genomic mapping and alignment program for mRNA and EST sequences. Bioinformatics 2005, 21(9):1859-1875. 
42. Wu TD, Nacu S: Fast and SNP-tolerant detection of complex variants and splicing in short reads. Bioinformatics, 26(7):873-881.

43. Li H, Handsaker B, Wysoker A, Fennell T, Ruan J, Homer N, Marth G, Abecasis G, Durbin R: The Sequence Alignment/Map format and SAMtools. Bioinformatics 2009, 25(16):2078-2079.

44. Trapnell C, Williams BA, Pertea G, Mortazavi A, Kwan G, van Baren MJ, Salzberg SL, Wold BJ, Pachter $\mathrm{L}$ : Transcript assembly and quantification by RNA-Seq reveals unannotated transcripts and isoform switching during cell differentiation. Nat Biotechnol 2010, 28(5):511-515.

45. Katz Y, Wang ET, Airoldi EM, Burge CB: Analysis and design of RNA sequencing experiments for identifying isoform regulation. Nat Methods 2010, 7(12):1009-1015.

46. Maere S, Heymans K, Kuiper M: BiNGO: a Cytoscape plugin to assess overrepresentation of gene ontology categories in biological networks. Bioinformatics 2005, 21(16):3448-3449.

47. Merico D, Isserlin R, Stueker O, Emili A, Bader GD: Enrichment map: a network-based method for geneset enrichment visualization and interpretation. PLoS One 2010, 5(11):e13984.

\section{Figures}


Fig. 1

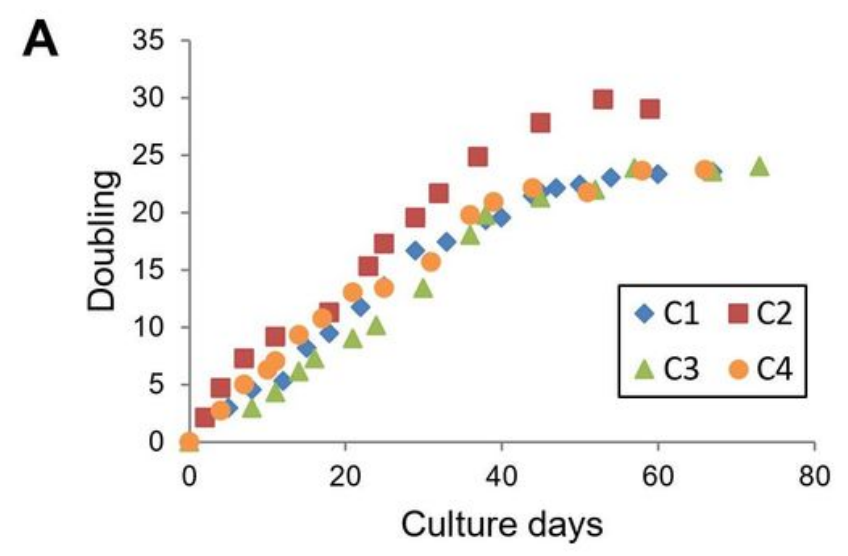

C

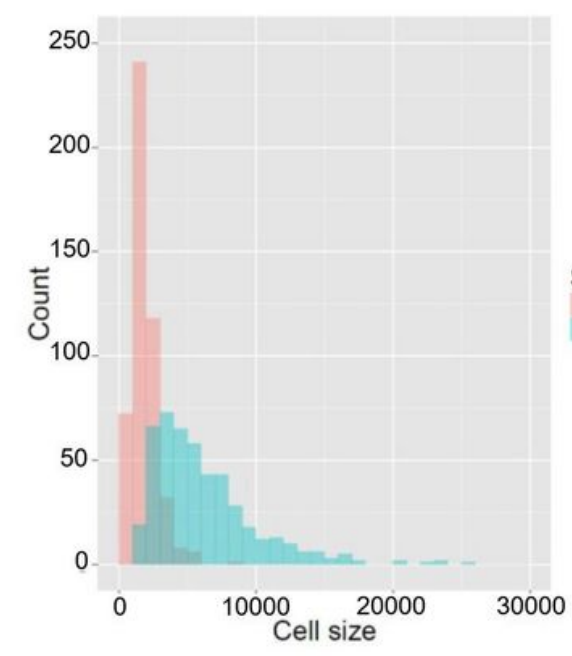

B

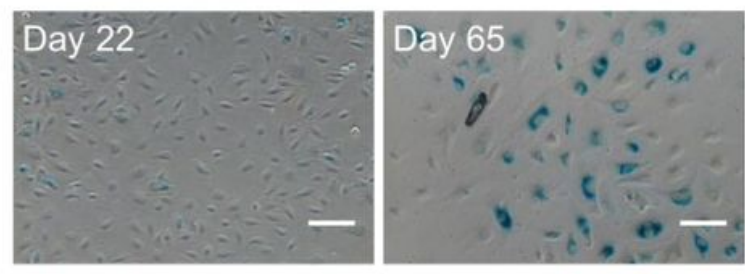

D

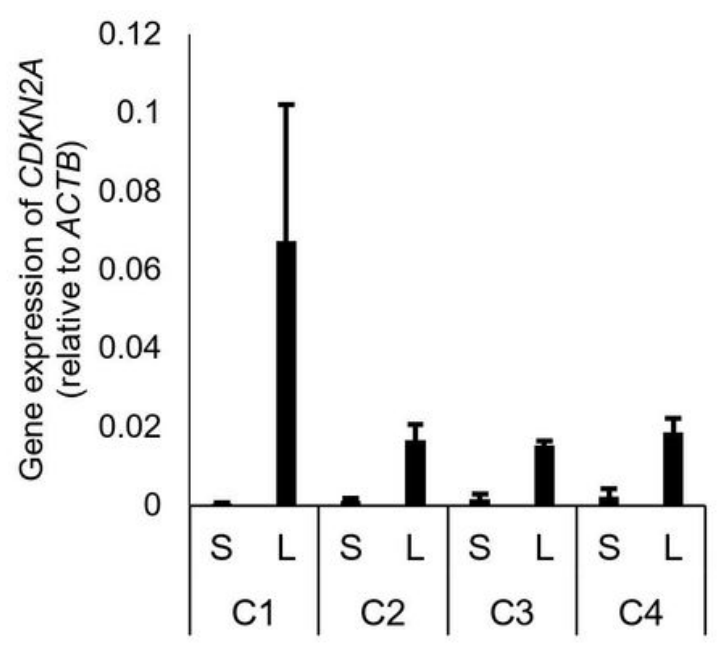

\section{Figure 1}

Replicative senescence of HUVEC (A) The doubling rate of HUVECs during replicative senescence. The Xaxis indicates culture days. The Y-axis indicates the population doubling (PD) rate. (B) Representative pictures of SA- $\beta$-Gal-stained HUVECs. C1 clone of HUVECs cultured for 22 days (left) or 65 days (right). Scale bar $=100 \mu \mathrm{m}$. (C) The cellular size of HUVECs during replicative senescence. The Y-axis indicates the number of HUVECs. The X-axis indicates the cell body mass. Pink and blue bars represent HUVECs cultured for 24 and 66 days, respectively. (D) The qPCR analysis for the expression of CDKN2A of HUVECs during senescence. The $Y$-axis indicates the relative expression level of CDKN2A in four individual clones of HUVECs. The X-axis indicates the time of sampling. $S$ and $L$ indicate short and long culture periods (e.g., day 22 and day 65), respectively. $\beta$-actin was used as an internal control. Data represent mean \pm standard deviation (SD) of three independent experiments. 
Fig. 2

A

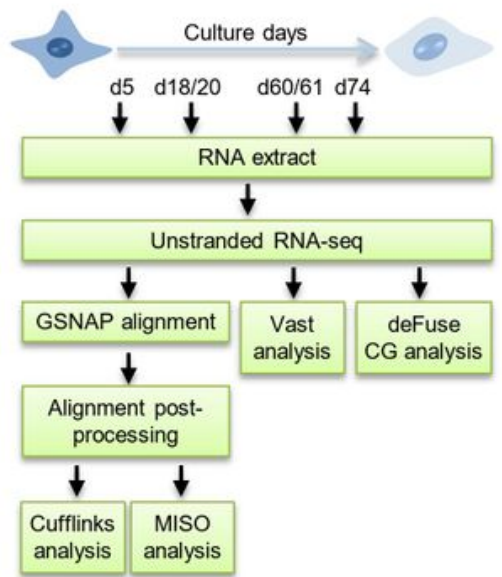

B

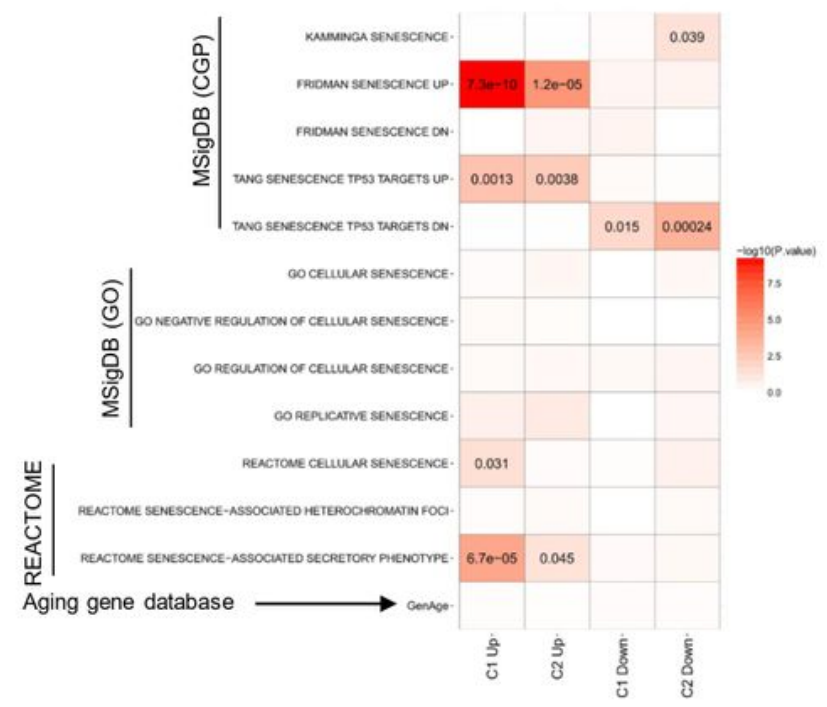

C
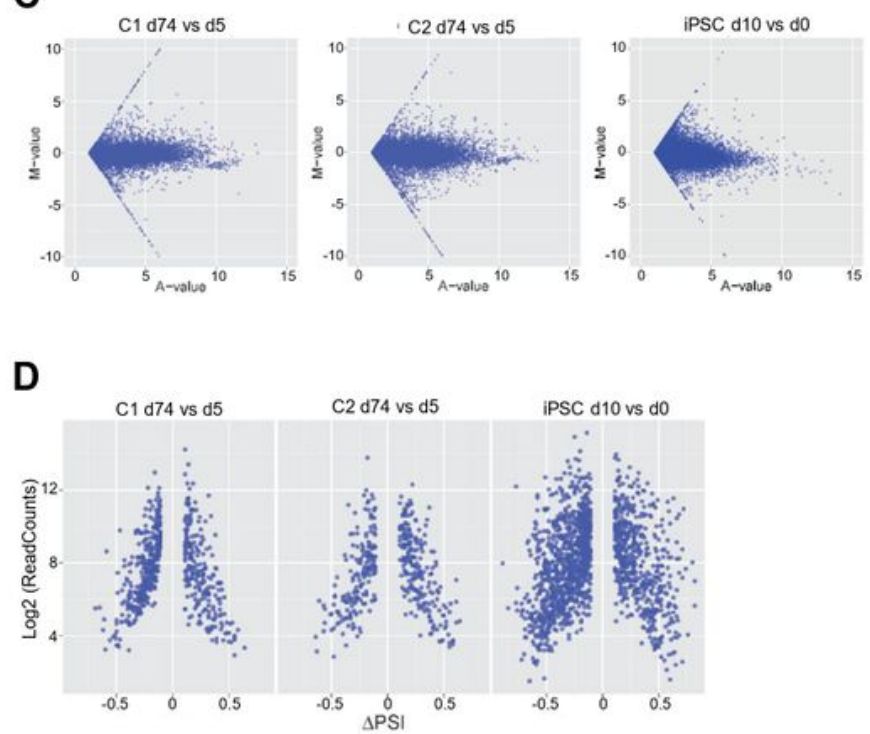

E

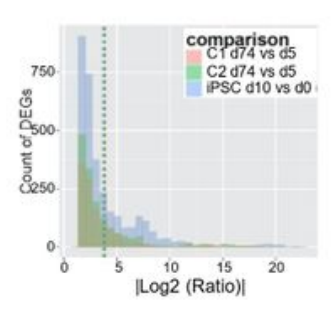

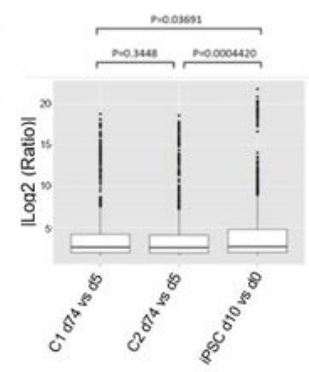

$\mathbf{F}$

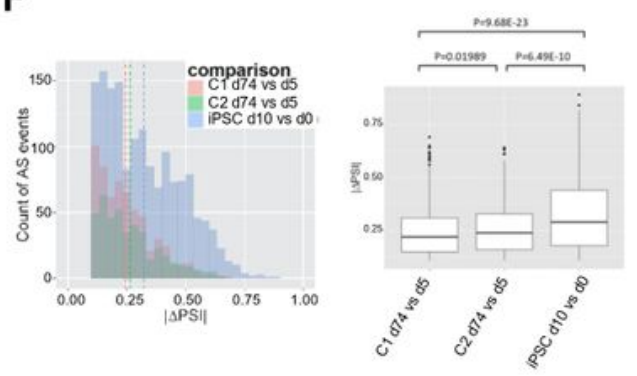

\section{Figure 2}

Analysis of DEGs and splicing alteration induced by senescence in HUVECs (A) Flowchart showing the analysis of RNA-seq data. (B) Heat map showing the P-values of enrichment analysis of genes differentially expressed between days 5 and 74 in $\mathrm{C} 1$ and $\mathrm{C} 2$ clones against known senescence gene sets. (C) MA plots of expression profiles of three data sets. The X-axis indicates the FPKM values, and the Y-axis indicates the log2 scale ratio of FPKM in HUVECs of clones C1 (left) and C2 (middle) cultured 
between 5 and 74 days, respectively, and neuronal differentiation from iPSCs between 0 and 10 days (right). (D) Volcano plots of AS events in HUVECs of clones C1 (left) and C2 (middle) cultured for 5 and 74 days and neuronal differentiation from iPSCs for 0 and 10 days (right). The $X$-axis indicates the $\triangle P S I$ value, and the $\mathrm{Y}$-axis indicates the log2-transformed values of read counts assigned for each splicing event. (E) The distribution of DEGs in $\mathrm{C} 1$ and $\mathrm{C} 2$ cultured between 5 and 74 days, respectively, and in neuronal differentiation of iPSCs cultured between 0 and 10 days (left). The $Y$-axis indicates the number of DEGs, and the $X$-axis indicates the log2 ratio. The dotted line shows the mean log2 ratio. Pink, green, and blue colors represent $\mathrm{C} 1, \mathrm{C} 2$, and iPSCs, respectively: Box plot of the absolute value of the log2 ratio FPKM for HUVECs in clones $\mathrm{C} 1$ and $\mathrm{C} 2$ cultured between 5 and 74 days, respectively, and in neuronal differentiation of iPSCs cultured between 0 and 10 days (right). The P-value was calculated using the Wilcoxon test. (F) The distribution of AS events in $\mathrm{C} 1$ and $\mathrm{C} 2$ between 5- and 74-days culture, respectively, and in iPSCs between 0 - and 10-days culture (left). The Y-axis indicates the number of AS events, and the $\mathrm{X}$-axis indicates the absolute value of $\triangle \mathrm{PSI}$. The dotted line shows the mean value of $\triangle \mathrm{PSI}$. Pink, green, and blue colors represent $\mathrm{C} 1, \mathrm{C} 2$, and iPSCs, respectively. Box plot of the absolute value of $\triangle \mathrm{PSI}$. log2 ratio FPKM for HUVECs in clones $\mathrm{C} 1$ and $\mathrm{C} 2$ between 5- and 74-days culture, respectively, and in neuronal differentiation of iPSCs between 0 and 10 days (right). The P-value was calculated using the Wilcoxon test. 
Fig. 3

A

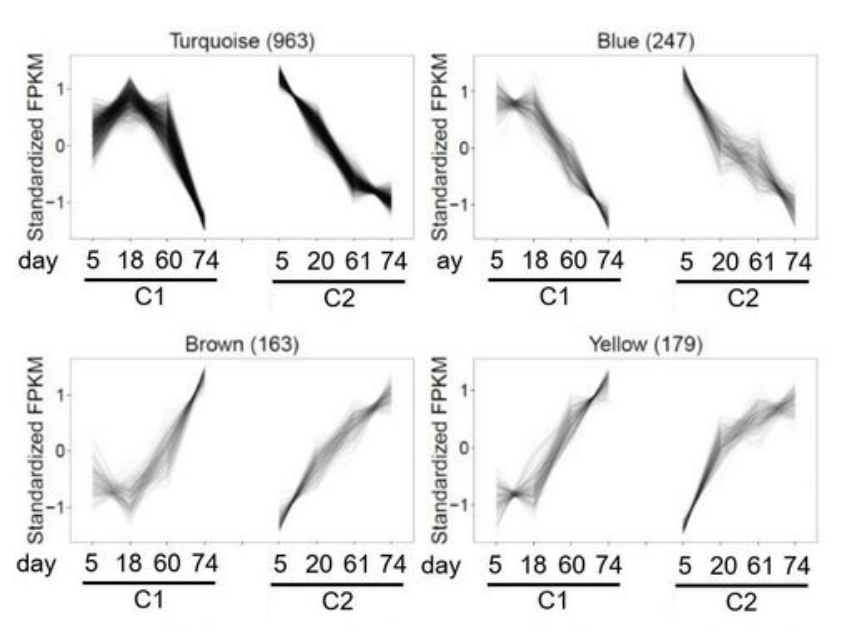

B

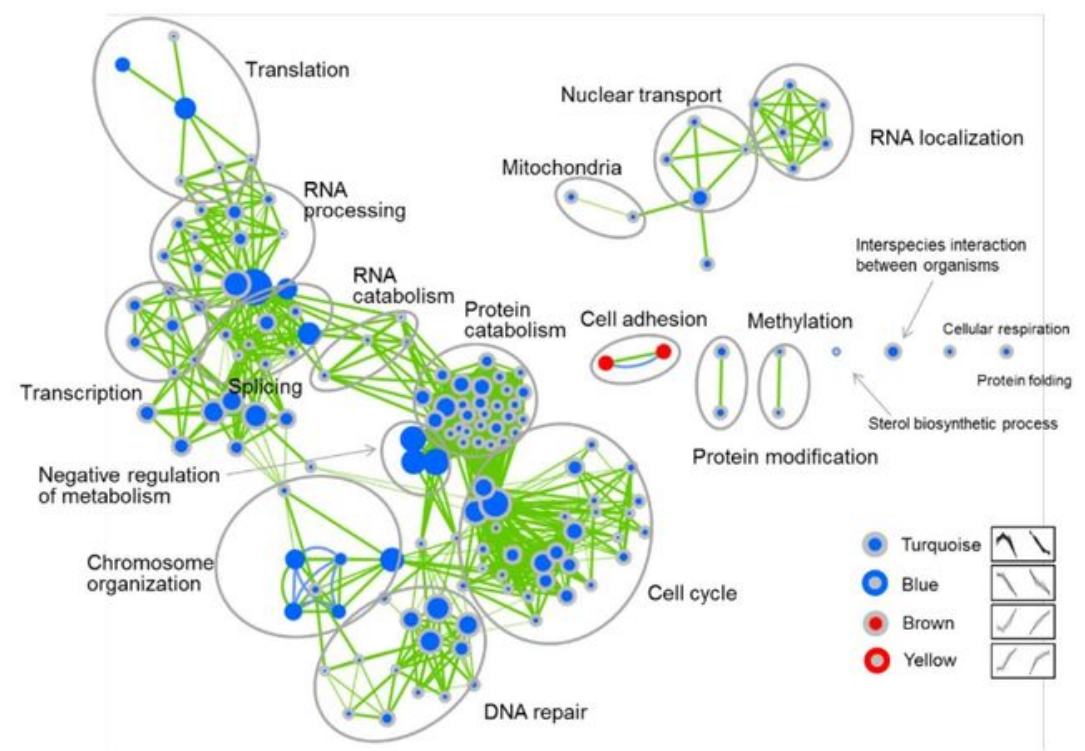

C

\begin{tabular}{|l|l|l|l|}
\hline Module & IPA upstream analysis & $\begin{array}{l}\text { TFBSs upstream } \\
\text { analysis }\end{array}$ & $\begin{array}{l}\text { Gene included in each } \\
\text { module }\end{array}$ \\
\hline Turquoise & $\begin{array}{l}\text { E2F4, E2F1, HNF4A, } \\
\text { CDKN1A, TBX2, RB1, } \\
\text { CDKN2A, MYC }\end{array}$ & $\begin{array}{l}\text { E2F1, E2F4, NRF1, } \\
\text { ELK1, SP1, MYC }\end{array}$ & $\begin{array}{l}\text { E2F1, E2F4, SIRT6, NRF1, } \\
\text { SREBF1, TGFB1, ELK1, HRAS, } \\
\text { FN1 }\end{array}$ \\
\hline Blue & $\begin{array}{l}\text { SCAP, SREBF2, } \\
\text { SREBF1, SIRT2, INS1, } \\
\text { INSIG1, SIRT6 }\end{array}$ & $\begin{array}{l}\text { SP1, YY1, EGR1, } \\
\text { MYC }\end{array}$ & $\begin{array}{l}\text { SP1, SREBF2, INSIG1, MYC, } \\
\text { YY1, RB1 }\end{array}$ \\
\hline Brown & $\begin{array}{l}\text { NKX2-3, IFNG, MYCN, } \\
\text { ESR1, TFEB, PRL, } \\
\text { HRAS }\end{array}$ & & ERBB2, CDKN2A \\
\hline Yellow & $\begin{array}{l}\text { TFEB, ERBB2, TGFB1, } \\
\text { FN1, HGF, HRAS, } \\
\text { ITGB2 }\end{array}$ & SREBP1, AP1 & SIRT2, CDKN1A, ITGB2 \\
\hline & & & \\
\hline & & & \\
\hline
\end{tabular}

\section{Figure 3}

WGCNA of genes showing monotonic decrease or increase in expression (FPKM values) during HUVEC senescence (A) WGCNA of the expression patterns of hub genes in the major co-expressed clusters, based on FPKM values in HUVECs cultured for different durations. The Y-axis indicates the standardized FPKM value. (B) Biological process enrichment map of DEGs involved in the culture period-dependent down-regulated (turquoise and blue) and up-regulated (brown and yellow) clusters. Each node represents 
a GO biological process gene set. The blue circle indicates biological processes enriched among downregulated genes, and the red circle indicates biological processes enriched among up-regulated genes. Color intensity indicates statistical significance. (C) Predicted regulatory network during HUVEC senescence. The first column represents the module name; the second and third columns represent each gene symbol by the IPA upstream analysis and TFBS upstream analysis; the fourth column represents the gene symbol included in each module.

\section{Fig. 4}

A

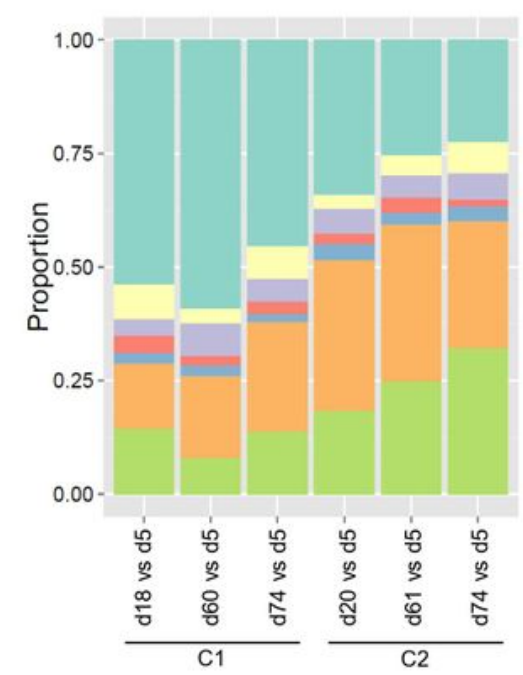

B

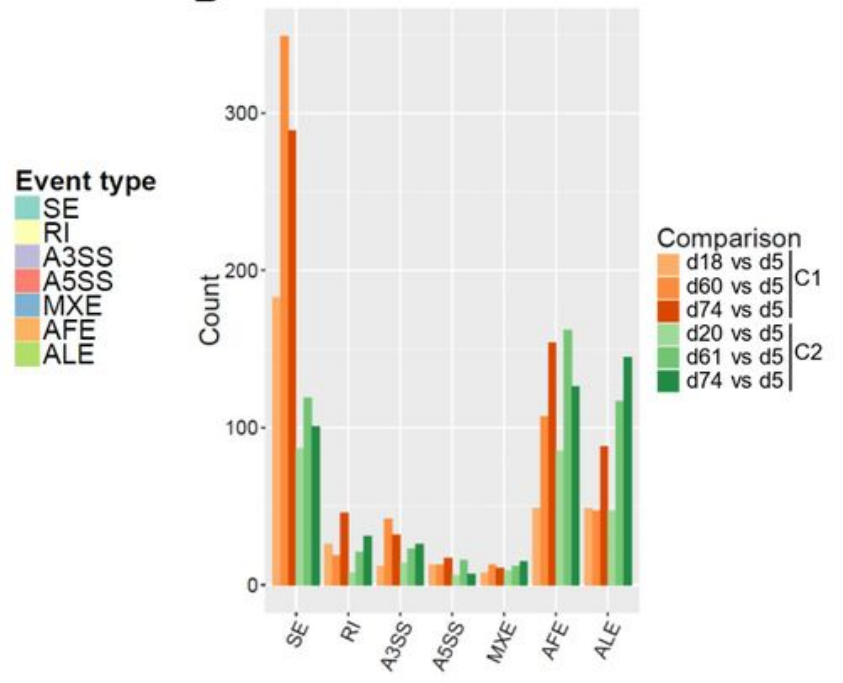

C

AS events (d5 vs d72)

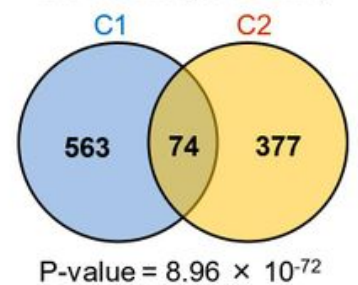

E

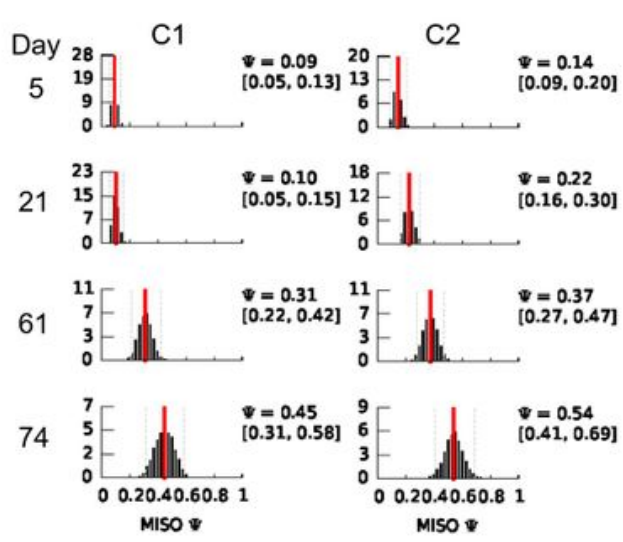

F

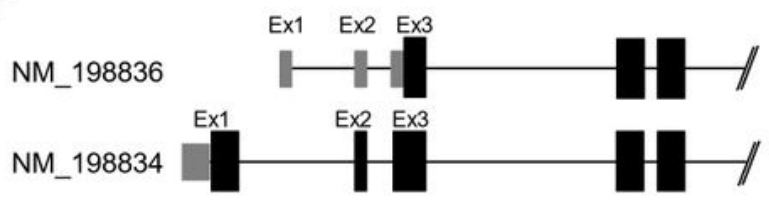

G

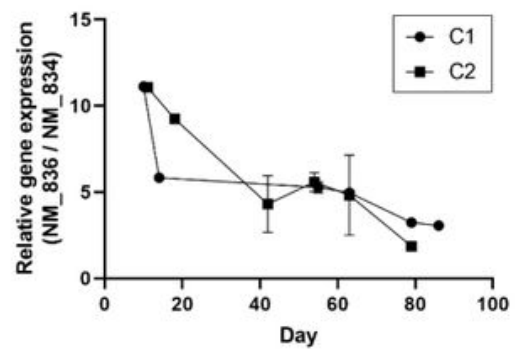


Predominant changes in AFE- and ALE-type splicing during HUVEC senescence (A) Proportions of different types of splicing events in HUVECs in each culture period, as determined by MISO analysis. (B) Splicing event type counts for each comparison. (C) Venn diagram showing the overlap between AS events between day 5 and day 74 in $\mathrm{C} 1$ and $\mathrm{C} 2$ clones. Statistical significance was determined using Fisher's exact test. (D) List of alternatively spiced genes related to senescence. (E) The $\triangle P S I$ distribution for the splicing of ACACA in $\mathrm{C} 1$ and $\mathrm{C} 2$ clones during senescence. The $\mathrm{Y}$-axis indicates the FPKM values, and the X-axis indicates the MISO PSI posterior distribution in both $\mathrm{C} 1$ (left) and C2 (right) at the indicated culture condition. (F) Schematic representation of two spliced isoforms, NM_198836 and NM_198834, of ACACA mRNA. Light-colored boxes represent untranslated exons, and dark-colored boxes represent translated exons. Lines represent introns. (G) Analysis of ACACA gene expression by qPCR. The $\mathrm{Y}$-axis indicates relative gene expression (NM_198836 [NM_836]/NM_198834 [NM_834]). The X-axis indicates culture days. Data represent mean \pm SD of two independent experiments. 
Fig. 5

A

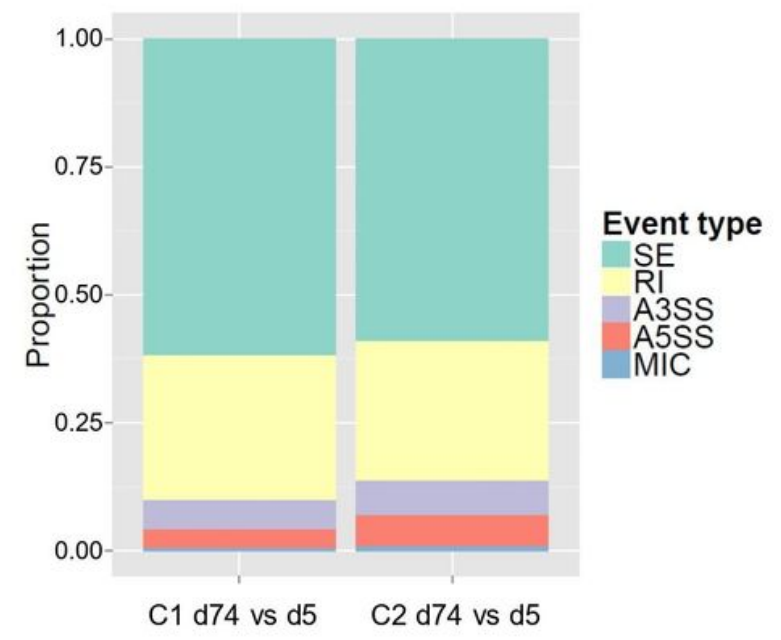

C

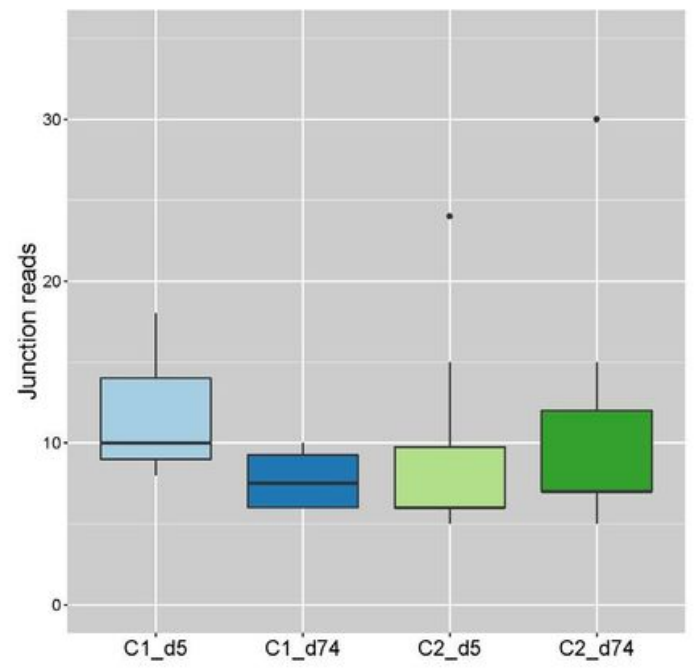

B
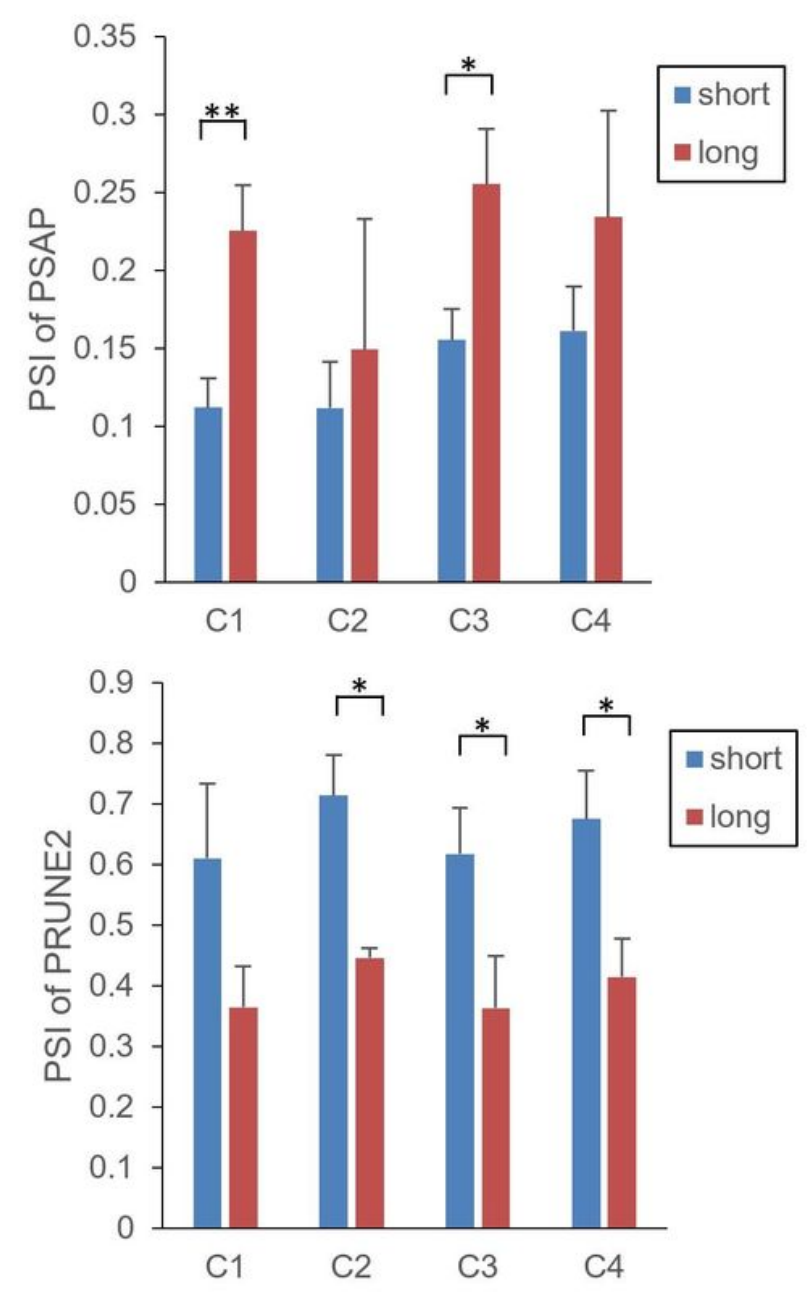

Figure 5

Microexon alterations during HUVECs senescence (A) Proportions of different types of splicing events in HUVECs in each culture period, as determined by VAST-TOOLS. (B) RT-PCR assays monitoring AS patterns of microexons in PSAP (top) and PRUNE2 (bottom) in HUVECs under short and long culture periods. PSI values are shown in the right bar plot. (C) Box plots showing the distribution of conjoined gene junction reads per condition. Data represent mean $\pm S D$ of three independent experiments $(* P<0.05, * \star P<0.01$; Student's t-test). 
Fig. 6

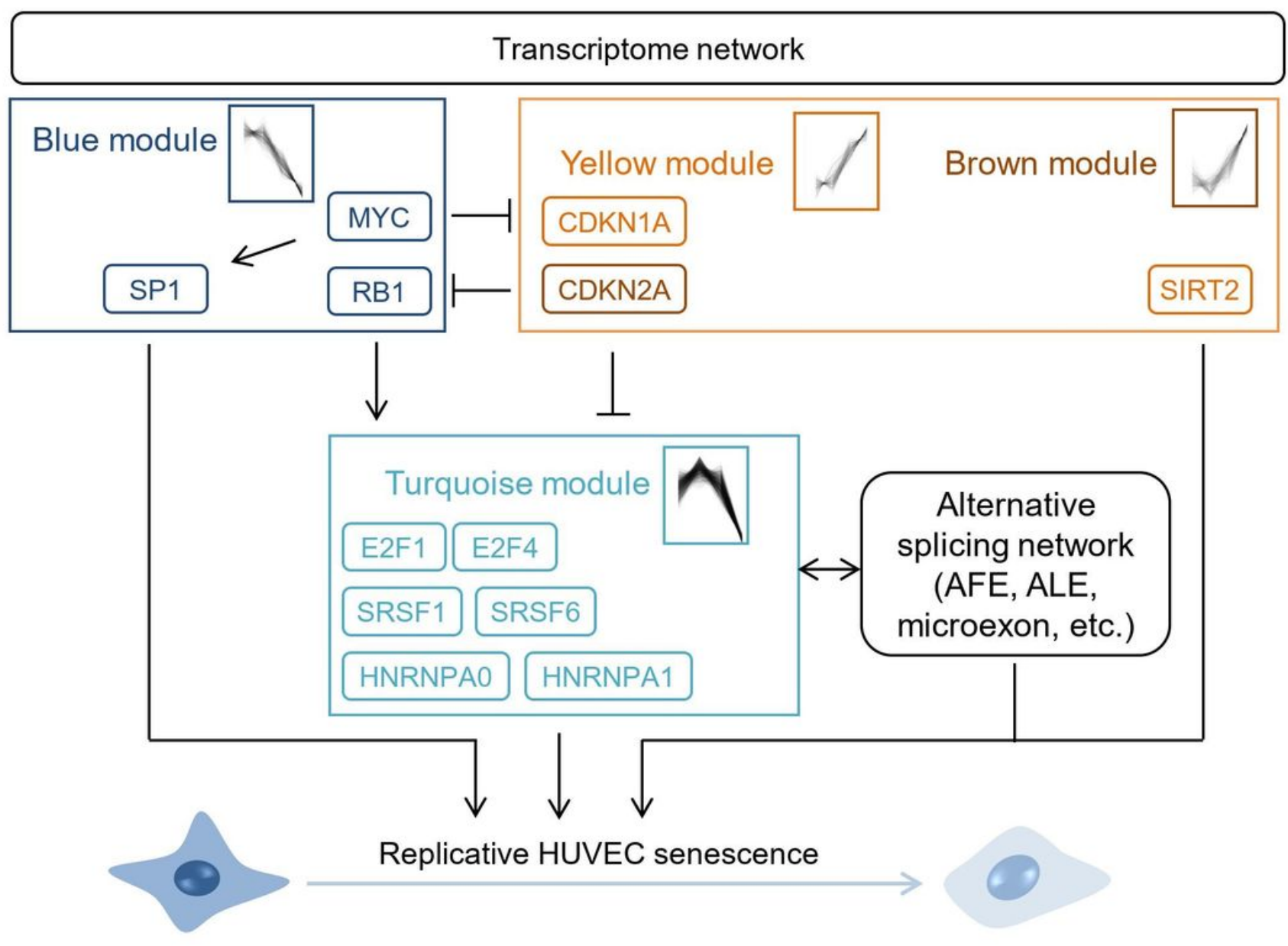

Figure 6

Predicted regulatory network that controls HUVEC senescence

\section{Supplementary Files}

This is a list of supplementary files associated with this preprint. Click to download.

- OhorietalSupplFigf.pdf

- TableS1.xlsx

- Tables2.xIsx

- TableS3.xlsx 